\title{
The First Case of Asymptomatic Infection of COVID-19 - Kashgar Prefecture, Xinjiang Uygur Autonomous Region, China, October, 2020
}

\author{
Ji Wang, ${ }^{1, x}$; Jun Zhao ${ }^{2,8}$; Xiang Zhao'; Zhenguo $\mathrm{Gao}^{2}$; Yang Song'; Xin $\mathrm{Ma}^{2}$; \\ Kai Nie'; Aimin $\mathrm{Xu}^{3}$; Mahemuti ${ }^{2}$; Ling Zhang ${ }^{2}$; Wenbo $\mathrm{Xu}^{1, *}$; Yan $\mathrm{Cui}^{2, *}$
}

On October 24, 2020, a 17-year-old female was detected as having an asymptomatic infection of coronavirus disease 2019 (COVID-19) during a regular nucleic acid test in Kashgar Prefecture, Xinjiang Uygur Autonomous Region. She was then transferred to a local designated hospital for medical observation. The patient lived in Shufu County of Kashgar Prefecture and worked in a local garment factory. On the afternoon of October 24 after she was diagnosed as positive, a total of 831 persons in the garment factory were tested and the results were all negative. Afterwards, 16 close contacts and 406 of close contacts of close contacts were also tested, and 137 were positive and had asymptomatic infections.

On October 26, genome sequencing was performed at Xinjiang CDC using the Ion S5 (Thermo Fisher) and MinION (Oxford Nanopore) platform guided by experts of China CDC. Compared with the sequence of the Wuhan reference strain (NC_045512) (1), the whole genome sequence of the first asymptomatic infection of COVID-19 case had 23 nucleotide site mutations. Among them, 6 nucleotide mutation sites (C241T, C3037T, C14408T, A23403G, C18877T, G25563T) were characteristic of the European branch of the European family of the L genotype II.3 (Figure 1). The virus sequence had 3 additional nucleotide mutation sites (C2113T, C7765T, C17690T). The above 9 nucleotide mutation sites belong to the branch of B.1.9.

In addition, the virus sequence had 14 unique nucleotide mutation sites (T433C, C1415T, G8653T, C9891T, A13483G, G13920T, G16852T, G17658T, G17686T, T23978C, C25460T, G25912T,
G26426T, C28887T), which were not retrieved from the published global new coronavirus genome database and no sequence with more than two of these nucleotide mutation sites was retrieved. This suggested that these 14 mutation sites were characteristic of the asymptomatic infection case. The sequencing results showed that the genotype of the first asymptomatic infection case was different from that which had affected China and was not the spread from the reemergent cases in Xinjiang in July (2). The source of the virus was not a new "spillover" from the host or intermediate host.

The source of the COVID-19 virus has not yet been determined, and further investigation and large-scale nucleic acid testing are still being conducted.

doi: $10.46234 / \mathrm{ccdcw} 2020.233$

\# Corresponding authors: Wenbo Xu, xuwb@ivdc.chinacdc.cn; Yan Cui,cy8565603@sina.com.

\footnotetext{
National Institute for Viral Disease Control and Prevention, China CDC, Beijing, China; ${ }^{2}$ The Center for Disease Control and Prevention of Xinjiang Uygur Autonomous Region, Xinjiang, China; ${ }^{3}$ The First People's Hospital of Kashgar, Kashgar Prefecture, Xinjiang, China.

\& Joint first authors.
}

Submitted: October 28, 2020; Accepted: November 04, 2020

\section{REFERENCES}

1. Wu F, Zhao S, Yu B, Chen YM, Wang W, Song ZG, et al. A new coronavirus associated with human respiratory disease in China. Nature 2020;579(7798):265 - 9. http://dx.doi.org/10.1038/s41586-020-2008-3.

2. Chen C, Ma HMT, Jia ZY, Zhao X, Wang DY, Zhao J, et al. Reemergent cases of COVID-19-Xinjiang Uygur autonomous Region, China, July 16, 2020. China CDC Wkly 2020;2(39):761-3. http://dx.doi.org/10.46234/ccdcw2020.206. 


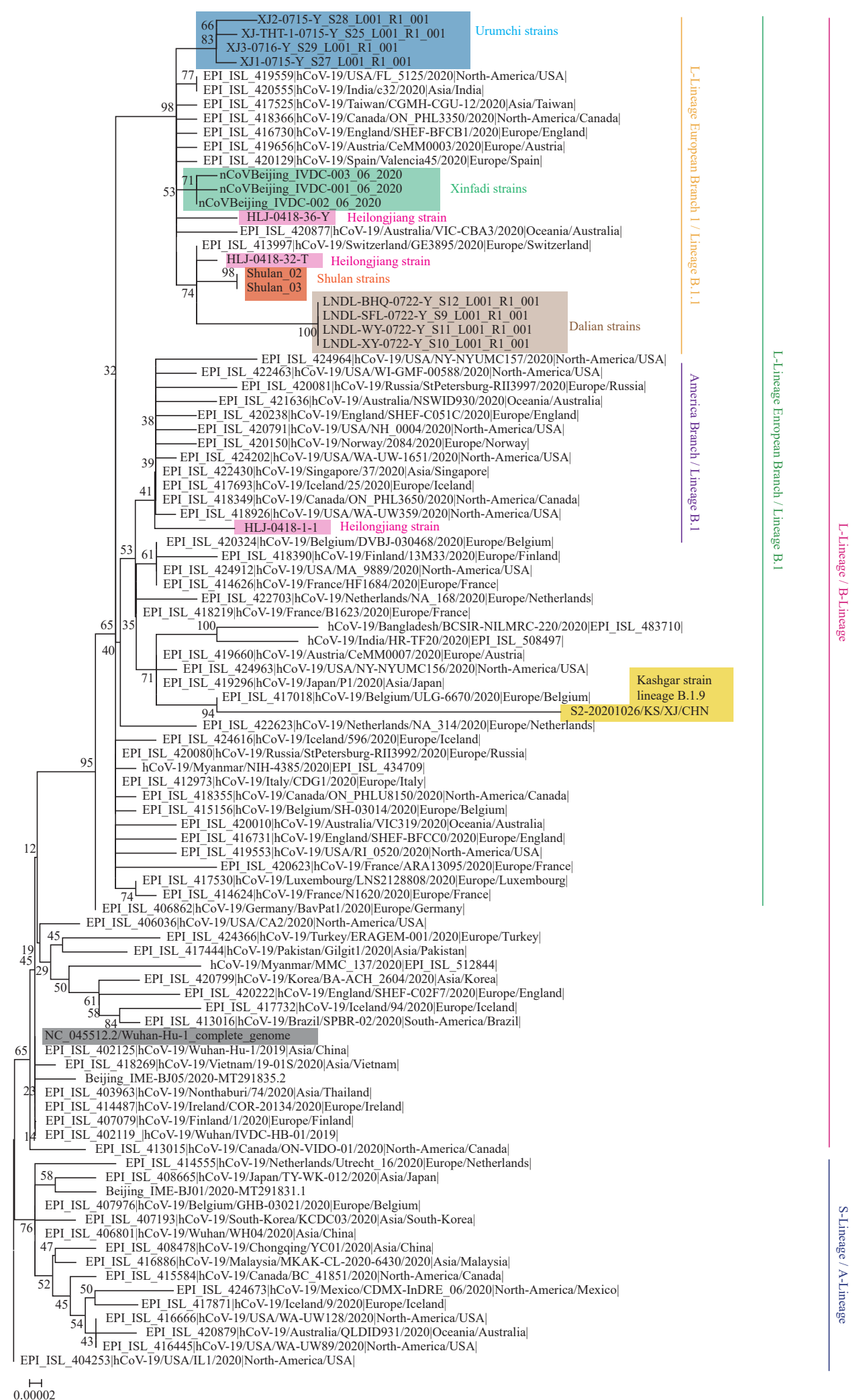

FIGURE 1. Phylogenetic tree based on the full-length genome sequences of the COVID-19 virus. The strains associated with specific outbreaks are as follows: Kashgar Prefecture (yellow); Urumchi (blue); Wuhan City in December 2019 (gray); Beijing Municipality Xinfadi Wholesale Market (green); northeastern China including Shulan and Heilongjiang Province related to imported cases (orange and violet, respectively); and Dalian City (brown). The $S(A)$ - or L(B)-lineage and sublineages of the COVID-19 virus were marked and colored on the right. 